\title{
CALAVIA SÁEZ Oscar, O Nome e o Tempo dos Yaminawa (etnologia e história dos Yaminawa do rio Acre)
}

\section{Pedro de Niemeyer Cesarino}

Traducteur : Philippe Erikson

\section{OpenEdition}

\section{Journals}

Édition électronique

URL : https://journals.openedition.org/jsa/10815

DOI : 10.4000/jsa. 10815

ISSN : $1957-7842$

\section{Éditeur}

Société des américanistes

Édition imprimée

Date de publication : 23 juillet 2009

Pagination : 237-243

ISSN : 0037-9174

Référence électronique

Pedro de Niemeyer Cesarino, " calavia sáez Oscar, O Nome e o Tempo dos Yaminawa (etnologia e história dos Yaminawa do rio Acre)», Journal de la Société des américanistes [En ligne], 95-1 | 2009, mis en ligne le 10 juillet 2009, consulté le 04 septembre 2022. URL : http://journals.openedition.org/jsa/ 10815 ; DOI : https://doi.org/10.4000/jsa.10815

Ce document a été généré automatiquement le 4 septembre 2022.

Tous droits réservés 


\title{
CALAVIA SÁEZ Oscar, O Nome e o Tempo dos Yaminawa (etnologia e história dos Yaminawa do rio Acre)
}

\author{
Pedro de Niemeyer Cesarino \\ Traduction : Philippe Erikson
}

\section{RÉFÉRENCE}

CALAVIA SÁEZ Oscar, o Nome e o Tempo dos Yaminawa (etnologia e história dos Yaminawa do rio Acre), Editora Unesp/Instituto Socioambiental, col. « Núcleo de Transformações Indígenas », São Paulo, 2006

o Nome e o Tempo dos Yaminawa est une version remaniée de la thèse de doctorat qu'Oscar Calavia Sáez a préparée, puis soutenue dans le cadre du Núcleo de História Indígena e Indigenismo de l'Universidade de São Paulo (NHII/USP). Cette étude ethnographique enrichit considérablement la littérature déjà fort abondante consacrée aux locuteurs de langues pano et, plus généralement, aux Amérindiens d'Amazonie occidentale. L'auteur se propose, avec ce livre sur les Yaminawa du rio Acre (Brésil), de nous livrer une ethnohistoire qui fasse ressortir, pour reprendre ses propres termes, des « proto-modèles ou de l'entropie» (p.15). Il nous offre de fait " une description systématique de cette supposée entropie " des Yaminawa, qui semblent en quelque sorte " hostiles à toute forme d'ordre, tant civique que logique» (p. 16). Mais Calavia Sáez reconnaît aussi les difficultés rencontrées avant d'obtenir suffisamment de données approfondies et systématiques: vicissitudes sans doute inhérentes au métier d'ethnographe. L'auteur inscrit en définitive son étude dans le champ des logiques de transformations auxquelles semblent confrontés des Yaminawa supposément «acculturés», ce qui justifie la publication de ce travail dans la collection Núcleo Transformações Indígenas (NuTI), dirigée par Eduardo Viveiros de Castro. 
2 D'après Calavia Sáez lui-même, son œuvre s'inscrit dans la lignée des travaux que Graham Townsley avait jadis consacrés à ce même peuple. Dès les toutes premières pages du livre, il montre dans quelle mesure ses analyses corroborent celles de son prédécesseur. Il en résulte un portrait des Yaminawa comme "parfait exemple de la fécondité et de la priorité attribuées à alter dans la définition d'un modèle de sociabilité “amérindien" " (p. 28). Il souligne également « certains aspects de leur chamanisme qui remettent en question la perception de telles pratiques comme "rituelles" ou "traditionnelles" " (ibid.). Toujours dans les pages initiales, Calavia Sáez revient sur la complexité du processus qui aboutit à la désignation de l'ethnie en question au moyen du terme "Yaminawa », précisant que: "un nom qui évolue en se modifiant [...] est fatalement un nom ambigu, mais son fractionnement diachronique - ce qu'on propose de faire ici est de l'histoire - permet de rechercher l'ordre sous-jacent à cette ambiguïté » (p. 30).

De sa présentation du système de parenté ressort la complexité de la notion de yura ( " corps » ou « chair », mais aussi " parent »), dont le sens ne s'explicite qu'à la lumière du contraste avec nawa, «l'étranger, ou l'extérieur en général» (p. 84). Calavia Sáez constate que " ces deux termes admettent des acceptions conjointes et situationnelles » (ibid.), coupant ainsi court à d'éventuelles interprétations substantivistes de ces catégories qui constituent un des noyaux essentiels (et paradoxaux) de diverses sociétés pano. « Nawa est une sorte de suffixe utilisé pour définir, et ainsi objectiver, des groupes humains» (p. 85), bien qu'il soit aussi utilisé pour désigner « une des moitiés de la partition du monde yura : la moitié correspondant à la part "extérieure" de l'intérieur " (ibid.). L'auteur souligne également la place de la notion de yurautsa, dont bien des équivalents existent chez d'autres peuples amérindiens, et qui désigne « un autre similaire, mais indéfini » (p. 86), "qui tend vers l'angle mort, et qui, en tant que substantif, dénote la non-relation, mais en tant que modificateur, bouleverse les classifications » (p. 87).

4 Calavia Sáez met l'accent sur le rôle du chef yaminawa comme «noyau d'attraction qui induit de nombreux groupes familiaux à s'installer auprès de lui» (p. 89), contrebalançant ainsi la tendance à la dispersion et au décentrement qui caractérise cette société. La citation suivante illustre bien ce thème, qui fait en somme office de fil conducteur: «[...] la chefferie est un point d'observation privilégié qui permet d'entrevoir comme une unité quelque chose qui, vu de plus près, apparait comme un agrégat sans ordre ». L'auteur examine ensuite le système dravidien tel qu'il se présente chez les Yaminawa : « un système exhaustif qui, en principe, ne laisse personne échapper à ses mailles " (p.91), mais non sans offrir une inflexion particulière par le biais de l'usage de termes vocatifs, qui induisent « un tournant égocentrique, annulant la temporalité orientée du système dravidien et $\mathrm{y}$ substituant une classification perspectiviste, où l'énonciateur dédouble la grille à partir de sa position » (p. 97).

5 Ce n'est nullement par hasard que l'auteur annonce « une sorte de victoire du système "yura-nawa" sur le dravidianat, dans la mesure où ce sont ses contours particulièrement flous qui régissent les alliances plutôt que les catégories du second» (p. 106). Calavia Sáez lie la parenté à l'histoire, renforçant ainsi la thèse poursuivie tout au long du livre : «Toute une pragmatique de la terminologie de parenté qui, dans son ensemble, renvoie le cas yaminawa dans cet espace du "dravidianat concentrique", oxymore qu'on pourrait comprendre ici comme un des noyaux de l'histoire yaminawa » (p. 115). Voilà bien une affirmation stratégique, susceptible de remettre en question le fallacieux problème de 
l'acculturation par le biais du recours à l'histoire; cette dernière prenant tout son sens dès lors que l'on perçoit que «les ambiguïtés d'une culture gardent toujours la possibilité de reformulation du système, lorsque le besoin s'en fait sentir » (p. 116).

6 La description des fêtes yaminawa illustre leur caractère mimétique à l'égard des coutumes des Blancs (encore une constante présente chez tous les Pano, voire au-delà), et met en exergue l'importance du rôle centralisateur du chef qui organise les festivités. On y constate que: «le principal danger qui guette la société yaminawa n'est pas la discorde, mais la dissolution des liens » (p. 133). L'intention mimétique est notable dans les festivités marquées par "l'exo-orientation» caractéristique de la plupart des systèmes pano. La même tendance se retrouve dans les rituels d'invention récente : «Si la performance des rituels anciens s'élaborait à partir de substances et d'esprits de l'extérieur, dans les rituels traditionalistes, c'est à destination des autres que les sociétés indigènes se mettent en scène » (p.135), passant ainsi d'une logique centripète à sa symétrique, centrifuge.

7 Cette tendance à « l'exo-orientation », que l'on retrouve à toutes sortes de niveaux de la pensée et de la société yaminawa, caractérise également la figure du chef, «...qui manipule le curseur yura/nawa afin qu'il demeure le critère principal de la formation de la société »(p.148). Dans sa brève présentation du chamanisme yaminawa, auquel l'auteur affirme n'avoir pu obtenir qu'un accès limité, le décentrement s'avère une fois encore fondamental. Conformément à une métaphore assez répandue en Amazonie, les visions produites par l'ayahuasca, le shori, sont ainsi comparées à ce qu'on peut voir « à la ville» ou "à la télévision» (p.154). Calavia Sáez écarte ici encore tout risque d'essentialisation de ce type de chamanisme en affirmant que : «[...] ce complexe n'est pas une structure traditionnelle susceptible de perdurer ou de disparaitre, mais un mode, pour traditionnel qu'il soit, d'exploration de l'extérieur, contemporain des mutations actuelles et lui-même tout sauf immuable» (p.155). Une telle perspective permet à l'auteur d'affirmer, dans le sillage de Townsley, que le complexe du shori est « une science, une œuvre ouverte, plus ou moins occulte» (p. 164).

8 Muni de tout cet arsenal, l'auteur s'attaque à ce qui apparaît, à ses yeux, comme «le problème yaminawa », autrement dit, leur apparente " déculturation » et "anomie ", l'échec des projets de développement les concernant, « leur incapacité à évoluer dans le périmètre légal de leur territoire [...], leur incapacité à maintenir ou produire des signes distinctifs d'identité indigène et, au fond, leur anarchie» (p.173). Calavia Sáez n'en révèle pas moins que, même ainsi, les apparences occultent «la continuité d'une tradition » (p. 174), en raison de " la cohérence entre l'actualité yaminawa et leur passé » (p. 175). L'étude des premiers contacts et des relations successives (toutes marquées par l'échec) avec différents patrons, missionnaires et, plus tard, membres de la FUNAI et de diverses ONG, met au jour toute une série de déplacements, de flux migratoires, de reconfigurations et de fissions qui reflètent, chacune à sa manière, "le pluralisme sociologique du groupe» (p.180). Ce dernier résultant aussi, par certains aspects, des disputes et des vendettas que décrit l'auteur, selon qui : « [...] l'objectif des disputes est la division elle-même, d'où résultent en effet des groupes bien définis - qui, avant ou après, se mettent à fonctionner comme supports d'échanges de conjoints » (p. 187). Et voilà pourquoi « la guerre peut remplir un rôle structurant, susceptible de compléter ou de modifier celui de l'alliance » (ibid.).

S'appuyant sur un des récits qu'il a recueilli auprès de plusieurs narrateurs yaminawa qui lui racontaient, par fragments, des épisodes du temps des anciens en parallèle avec les 
temps actuels, Calavia Sáez affirme qu'il ne s'agit pas tant d'une réminiscence, au sens mémoriel, mais plutôt d'une histoire, «au plein sens du terme, avec toute l'intentionnalité et les critères de signification afférents» (p. 217). Un peu plus loin, l'auteur retrouvera encore cette même historicité dans les chants féminins yamayama, dont il écrit que : «l'histoire [y] est parenté; tout comme l'histoire [y] est infortune » (p. 222). La parenté comme histoire, autrement dit : « des récits, partant de points de vue particuliers, sur les transformations du modèle [...] ou sur les contradictions engendrées par sa pratique » (p. 6). À la limite, poursuit-il, « l'histoire est dissolution de la parenté » (p. 223), teintée de la mélancolie qui accompagne les réminiscences yaminawa (ibid.).

Et non sans raisons. L'auteur a compilé bon nombre de récits d'épisodes du boom du caoutchouc - avec son cortège de raids, massacres, soumissions et conflits entre Pano, tant il est vrai que " le boom n'a fait qu'amplifier des actes de prédation bien anciens " (p.229) - qui illustrent bien l'effet de kaléidoscope que l'appellation générique "Yaminawa ", tout au long de son (désastreux) parcours, a tant bien que mal réussi à occulter. Les différents moments et les différentes formes de relations établies, soit à travers l'exploitation et l'assujettissement menées par les Blancs, soit à travers les programmes officiels du SPI, étaient cependant caractérisés par ce qui semblait être une constante de la part des Yaminawa : « La segmentation des groupes relève d'une stratégie plus ou moins consciente face aux Blancs, qui vise à filtrer leur pouvoir, laissant passer les biens manufacturés tout en évitant les épidémies et la soumission » (p. 237).

11 L'auteur accorde la plus grande attention à la fameuse prolifération des noms qui admettent le suffixe -nawa : « les noms se perpétuent même après avoir perdu leur sens jusqu'à ce qu'ils le récupèrent » (p. 258). Comme un joker, le terme en vient alors à posséder des valeurs définies par ses différents emplois: "L'ethnonyme crée l'ethnie, parce que les groupes définis de l'extérieur, mais générés à partir de présupposés symboliques qu'ils partagent, tendent à se constituer en unités politiques de facto, et même à assumer certaines des caractéristiques différentielles qui leur ont été attribuées de l'extérieur » (p. 260). Ici, « les identités n'existent qu'au sein d'un système - de noms », offrant ainsi une certaine constance structurale à ce qui semblait a priori relever accidentellement $\mathrm{du}$ boom $\mathrm{du}$ caoutchouc, rendant ainsi possible une «vision nominaliste de l'ethnologie pano » (p. 260).

Peuple de la hache (yami signifie « hache »), les Yaminawa sont un maillon de ce vaste et complexe réseau de noms qui se retrouvent dans la plus grande diversité des époques et des situations, ce qui, note Calavia Sáez, suggère « soit de longs voyages, soit un groupe humain organisé à l'échelle géographique » (p. 271). Au sein de ce système, des relations de commerce, de guerre et de captivité rendent problématique la distinction entre peuples fluviaux et interfluviaux, autrement dit entre civilisés et sauvages. Les Shipibo (riverains) apparaissent pourvus d'une culture matérielle sophistiquée, mais, à l'occasion, n'en sont pas moins caractérisés par leur « aspect indéniablement sylvestre » (p. 276). Les redoutables Cashibo, pour leur part, passent par un processus inverse de "fluvialisation", en dépit de leur réputation d'incorrigibles anthropophages. "La répartition des niches écologiques ", conclut logiquement l'auteur, «ne traduit pas des différences culturelles, mais des disputes politiques ou guerrières» (p.276), et l'opposition fluvial/interfluvial doit être considérée comme une " catégorie opératoire de la pensée indigène » (p. 277). D'où l'affirmation suivante, qui constitue peut-être le point central de l'ouvrage : « Yaminawa ne désignait pas à l'origine une ethnie, mais une relation» (p. 277). 
13 C'est cette même quête des catégories indigènes qui, plus loin, conduira l'auteur à formuler l'avertissement suivant à propos des études sur l'Inca pano, marquées, une fois encore, par le substantialisme et "l'évolutionnisme résiduel »: « on rendrait un bien meilleur service à l'ethnohistoire indigène en démontrant plutôt la capacité de ces "historiens" d'adopter et d'adapter des informations plus récentes » (p. 286). L'Inca, tel qu'il apparaît dans les différents récits rencontrés ici ou là dans le bloc pano, « n'est rien de plus qu'un nom » (p. 295) ou, mieux, une notion utilisée par les sociétés pano dans le cadre de leur réflexion sur l'altérité : « le Je ne peut résulter que d'un Autre, aucune place n'étant laissée au monisme » (p. 302). L'Inca, dit-il, « nous éclaire sur l'utilité d'un nom dans une terre organisée par des pronoms sociologiques» (p. 305). Pour cette raison, le "point stratégique des structures sociales se déplace du centre (traditions, terminologies ou règles d'alliance) à la périphérie, à la frontière de l'identité ; c'est plutôt une manière de dire qu'elles sont le produit de l'histoire ou, plus concrètement, des historiens ou mythographes indigènes" (p.306). On peut ainsi échapper au dénigrement dont les mythes sont si souvent victimes: "Les transformations d'un ensemble mythique - ou d'un modèle social - sont des faits historiques, et pas uniquement des interprétations de faits historiques; elles peuvent se situer à la base ou aux avant-postes d'autres faits, plutôt que seulement derrière eux » (p. 308).

Et comment s'articulent les mythes au milieu des transformations? Comment les articuler à l'expérience de l'altérité qu'induisent les villes des Blancs par exemple? « On peut aisément s'apercevoir ici que la lecture des mythes d'un point de vue perspectiviste ne relève pas de la pure spéculation, à mille lieux de l'univers sublunaire de la raison pratique ou, en l'occurrence, de la politique indigéniste actuelle faite de conflits centrés sur des terres ou des ressources. La politique d'hyper-visibilité mise au point par les groupes indigènes les plus présents sur la scène multiculturelle ne s'inscrit guère dans la continuité de l'emphase sur l'impérieuse nécessité de se faire voir qui caractérise certaines cosmologies » (p.337). C'est peut-être le lien avec une telle cosmologie qui permet de mettre de côté le supposé problème de l'acculturation au regard de certains « échecs» des Yaminawa: "On interprète volontiers cet échec en évoquant une soidisant perte des repères traditionnels, mais je tends à croire qu'il résulte de causes précisément inverses : l'excès de fidélité envers une cosmologie [...]» (p. 360). C'est elle qui permet de comprendre le «territoire traditionnel », non pas à partir de coordonnées quantifiables, mais comme " un tracé aux contours variables qui va du yura au nawa» (ibid.), étant donné que «l'important réside dans cet espace sociologique, ou cosmologique, dans lequel les termes de l'identité et de l'altérité peuvent se dédoubler de manière satisfaisante » (p. 361).

$\mathrm{Au}$ bout du compte, cette constance des modèles socio-cosmiques - et des récits «mythiques» qui les développent - inciterait-elle à qualifier la société yaminawa de « froide» ou de "chaude»? Laissant de côté les diverses polémiques suscitées par le célèbre dilemme lévi-straussien, Calavia Sáez vise l'idiosyncrasie yaminawa: «[...] le sujet historique n'a pas besoin d'être révisé parce qu'il n'a jamais, pour autant que je sache, été réellement constitué » (p.373). Il n’y a pas d'identité rétroactive (chose qui, selon l'auteur, se retrouverait chez d'autres Pano) ; ce qu'on raconte n'est pas sa propre histoire, mais celle des autres (ibid.) : une histoire froide, peut-être, mais qui « coexiste sans difficulté avec une conscience du changement » (p. 374). L'affirmation selon laquelle «l'ethnographie et la fiction devraient se faire frontalières» (p.376), sur laquelle l'ouvrage tout à la fois s'ouvre et se referme, prend tout son sens à partir d'une certaine 
notion d'invention à travers laquelle se pense la construction du sens yaminawa. "Théoriciens ou commentateurs paresseux, les Yaminawa n'en sont pas moins des conteurs diligents: ils n'ont aucun discours méta-mythique à proposer, mais leurs mythes sont en soi de la métahistoire et de la métasociologie. En d'autres termes, leur histoire et leur sociologie vont jusqu'à englober la fiction, tandis que leurs mythes ne passent pas - et n'ont nul besoin de passer - pour de la fiction » (p. 377).

\section{AUTEURS}

\section{PEDRO DE NIEMEYER CESARINO}

Pós-doutorando na Universidade de São Paulo (DLCV/FFLCH/USP) 